\title{
Did we get lost in the Seventies? Adenoidectomy for middle ear disease in cleft palate children: a systematic review.
}

\author{
Cecilia Rosso ${ }^{1}$, Antonio Bulfamante ${ }^{2}$, Carlotta Pipolo ${ }^{3}$, Emanuela Fuccillo ${ }^{4}$, alberto \\ maccari $^{4}$, Paolo Lozza ${ }^{5}$, alberto scotti ${ }^{4}$, antonia pisani ${ }^{4}$, luca castellani ${ }^{4}$, giuseppe de \\ donato $^{4}$, Maria Chiara Tavilla ${ }^{4}$, Sara Portaleone ${ }^{5}$, Giovanni Felisati ${ }^{6}$, and alberto maria \\ saibene $^{4}$ \\ ${ }^{1}$ University of Milan \\ ${ }^{2}$ Università degli studi di milano \\ ${ }^{3}$ Università degli Studi di Milano \\ ${ }^{4}$ San Paolo Hospital - University of Milan \\ ${ }^{5}$ Azienda Socio Sanitaria Territoriale Santi Paolo e Carlo \\ ${ }^{6}$ University Clinic Milano, Polo San Paolo
}

February 14, 2021

\begin{abstract}
OBJECTIVES: Cleft palate children have a higher incidence of otitis media with effusion, more frequent recurrent acute otitis media episodes, and worse conductive hearing losses than non-cleft children. Nevertheless, data on adenoidectomy for middle ear disease in this patient group is scarce, since many feared worsening of velopharyngeal insufficiency after the procedure. This review aims at filling this knowledge gap by collecting the available evidence on this subject, to frame possible further areas of research and interventions. DESIGN: A PRISMA-compliant systematic review was performed. Multiple databases were searched with criteria designed to include all studies focusing on the role of adenoidectomy in treating middle ear disease in cleft palate children. After duplicate removal, abstract and full-text selection, and quality assessment, we reviewed eligible articles for clinical indications and outcomes. RESULTS: Among 321 unique citations, 3 studies were deemed eligible (2 case series and a retrospective cohort study). The outcomes were positive in all three articles in terms of conductive hearing loss improvement, recurrent otitis media episodes reduction, and effusive otitis media resolution (this last result being not statistically significant). CONCLUSION: Despite promising results, research on adenoidectomy in treating middle ear disease in the cleft population has stopped in the mid-Seventies. No data is therefore available on the role of modern conservative adenoidectomy techniques (endoscopic and/or partial) in this context. Prospective studies are required to define the role of adenoidectomy in cleft children, most interestingly in specific subgroups such as patients requiring re-tympanostomy, given their known risk of otologic sequelae.
\end{abstract}

TITLE: Did we get lost in the Seventies? Adenoidectomy for middle ear disease in cleft palate children: a systematic review.

\section{ABSTRACT}

OBJECTIVES: Cleft palate children have a higher incidence of otitis media with effusion, more frequent recurrent acute otitis media episodes, and worse conductive hearing losses than non-cleft children. Nevertheless, data on adenoidectomy for middle ear disease in this patient group is scarce, since many feared worsening of velopharyngeal insufficiency after the procedure. This review aims at filling this knowledge gap by collecting the available evidence on this subject, to frame possible further areas of research and interventions. DESIGN: A PRISMA-compliant systematic review was performed. Multiple databases were 
searched with criteria designed to include all studies focusing on the role of adenoidectomy in treating middle ear disease in cleft palate children. After duplicate removal, abstract and full-text selection, and quality assessment, we reviewed eligible articles for clinical indications and outcomes. RESULTS: Among 321 unique citations, 3 studies were deemed eligible ( 2 case series and a retrospective cohort study). The outcomes were positive in all three articles in terms of conductive hearing loss improvement, recurrent otitis media episodes reduction, and effusive otitis media resolution (this last result being not statistically significant). CONCLUSION: Despite promising results, research on adenoidectomy in treating middle ear disease in the cleft population has stopped in the mid-Seventies. No data is therefore available on the role of modern conservative adenoidectomy techniques (endoscopic and/or partial) in this context. Prospective studies are required to define the role of adenoidectomy in cleft children, most interestingly in specific subgroups such as patients requiring re-tympanostomy, given their known risk of otologic sequelae.

\section{KEY POINTS:}

- Cleft palate children have a higher incidence of middle ear disease compared to the general pediatric population

- Data on adenoidectomy for middle ear disease, diffusely available for the general pediatric population, is scarce in cleft palate children

- The scientific literature reports only 3 studies on adenoidectomy for middle ear disease in cleft palate children, all showing good outcomes

- Research on the role of adenoidectomy for middle ear disease in cleft palate children has not progressed further since the identified studies, which date back to the Seventies

- No available study on adenoidectomy for middle ear disease in cleft palate children validated more modern techniques such as partial, endoscopic and/or power assisted adenoidectomy in this patient group

Keywords: otitis media; hearing loss; cleft palate; cleft lip; adenoids;

Level of evidence: II Manuscript

\section{OBJECTIVE}

Children born with a nonsyndromic cleft palate, with or without cleft lip $(\mathrm{CP} \pm \mathrm{L})$, have a higher incidence of otitis media with effusion (OME), more frequent recurrent acute otitis media (RAOM) episodes, and worse early age OME-related conductive hearing losses (ORCHL) than non-cleft children[1-3]. With a wide variability among studies and age groups, $\mathrm{OME}$ in $\mathrm{CP} \pm \mathrm{L}$ children has been shown to reach incidences as high as $90 \%$ in the first year and $97 \%$ within the first 2 years of life[4].

There is a consensus among clinicians and studies that early ventilation tube insertion and early surgical cleft repair allow favorable results in this population [5]. Nevertheless, the role of repeated grommet insertion has been shown to correlate (albeit with a possible consistent selection bias) with a higher prevalence of chronic otitis media [5] in a population already at a higher incidence of re-tympanostomy when compared to non-cleft children.[6]

In the general pediatric population suffering from OME and RAOM there is conspicuous, albeit often lowlevel, evidence for the role of adenoidectomy and/or tympanostomy for OME, ORCHL, and RAOM. [7-9] Most specifically, adenoidectomy has proved beneficial in treating OME in the pediatric population, while its role in hearing thresholds and RAOM episodes is less defined. [7] Analogously, the role of tympanostomy for ORCHL is unclear and limited in time [8], while it appears moderately beneficial in reducing RAOM episodes [9]. NICE guidelines for example include adenoidectomy as a treatment option for OME [10], while the Italian Pediatric Otolaryngology society guidelines recommend adenoidectomy in carefully selected cases of OME and RAOM, with adenoiditis or Eustachian tube obstruction [11].

Conversely, data on adenoidectomy for middle ear disease in $\mathrm{CP} \pm \mathrm{L}$ children is scarce at best. Many authors discouraged the use of adenoidectomy in this population fearing worsening of velopharyngeal insufficiency 
[12], though endoscopy- and/or power-assisted modern techniques of adenoidectomy have proven safe also in this population[13].

This review aims at filling this knowledge gap by systematically collecting all the available evidence on the role of adenoidectomy in $\mathrm{CP} \pm \mathrm{L}$ in treating $\mathrm{OME}, \mathrm{RAOM}$, and ORCHL, to frame possible areas of further research and interventions.

\section{DESIGN}

This review protocol has been registered in the International Prospective Register of Systematic Reviews (PROSPERO) (ID CRD42021221115).

Search strategy

A systematic review was conducted between November 1 and December 31, 2020, according to the Preferred Reporting Items for Systematic Reviews and Meta-analyses (PRISMA) reporting guidelines ${ }^{13}$. We conducted systematic electronic searches for studies in the English, Italian, German, French or Spanish language reporting original data obtained from humans focused on the role of adenoidectomy in the treatment of middle ear disease in the cleft (lip and) palate population, with no publication date restrictions.

On November 18th, 2020, we searched the MEDLINE, Embase, Web of Science, Cochrane Library, and ClinicalTrials.gov databases with the following extremely wide search string (common to all databases):

("adenoidectomy" OR "adenotonsillectomy") AND "cleft"

We focused on studies on $\mathrm{CP} \pm \mathrm{L}$ children, diagnosed with $\mathrm{OME}, \mathrm{RAOM}$, and/or ORCHL, undergoing adenoidectomy or adenotonsillectomy. As per study design, we included only studies where the patients were diagnosed with a) OME, defined with tympanometry or otoscopy; b) ORCHL, quantified with pure tone audiometry; or c) RAOM. Similarly, another inclusion criterion for studies was reporting respective outcomes as a) resolution of OME, defined with normal tympanometry or otoscopy; b) improvement of ORCHL, quantified with pure tone audiometry; or c) reduction of acute otitis media episodes.

We excluded meta-analyses, systematic and narrative reviews, and case reports. There was no minimum study population size required. References from reviews and included articles were nevertheless checked for additional potentially relevant studies.

Abstracts and full texts were reviewed in duplicate (XX and XX, blinded for review purposes). All disagreements were resolved by evaluation from a third rater (XX, blinded for review purposes).

PICO criteria

The PICO criteria for the present review were as follows:

- Patients: Cleft palate or cleft lip and palate children with RAOM and/or OME - Intervention: Adenoidectomy (with or without tonsillectomy) - Comparison: Compared with no treatment for the condition . Outcome: Resolution of OME, improvement of ORCHL, or reduction of acute otitis media episodes

Data extraction and quality assessment

For each included article, we extracted the number of CLP patients treated, patients' age and sex, type of clefts included, clinical indications to adenoidectomy, type of clinical evaluation (for inclusion and/or outcomes), type of outcome(s) studied, and outcome(s).

Selected studies were assessed for both quality and methodological bias according to the National Heart, Lung, and Blood Institute Study Quality Assessment Tools (NHI-SQAT)[14]. Articles were rated in duplicate by two authors (XX and XX, blinded for review purposes) and disagreements were resolved by consensus. Items were rated as good if they fulfilled at least $80 \%$ of the items required by the NHI-SQAT, fair if they fulfilled between $50 \%$ and $80 \%$ of the items, and poor if they fulfilled less than $50 \%$ of the items, respectively. 
Also, the level of evidence was scored according to the Oxford Centre for Evidence-based Medicine (OCEBM) level of evidence guide[15].

\section{RESULTS}

\section{Search results}

The number of unique items retrieved from each database is available in Supplemental Table 1. Among the 321 unique research items initially identified, a total of 18 articles were selected for full-text evaluation and 7 further were retrieved from citations and reviews. Among the 25 articles undergoing full-text evaluation, 3 studies were retained for further analysis (see Supplemental Figure 1 for the selection process details and Supplementary Table 2 for details on the articles excluded during the full-text evaluation).

Two articles were case series[16,17] and one was a retrospective cohort study [18], all published from the mid-Sixties to the early Seventies. The resulting levels of evidence according to the OCEBM scale were rated 4 for two studies and 3 for the remaining one. According to the NHI-SQAT, all articles were judged of fair quality. Most articles lacked ample information to support the comparability of patients. No significant bias emerged from the evaluation of the articles. The pooled population from the three studies was 136 patients. Table 1 reports the characteristics of the included studies, their demographics, and the type of cleft included. Sex distribution among the samples was not reported in any of the articles.

Table 2 reports data in terms of procedures performed (adenoidectomy or adenotonsillectomy), indications to surgery, diagnostic and outcome assessment methods, and outcomes.

In one study all patients underwent adenotonsillectomy, in another, all patients underwent isolated adenoidectomy and, in a third study, patients underwent either adenoidectomy or adenotonsillectomy. Two out of three studies included patients with OMAR or ORCHL and one exclusively included patients with OME. The diagnosis was otoscopy-based in all studies but employed also audiometry for the two studies treating patients with ORCHL. In the single study on OME, the diagnosis was confirmed with myringotomy in all but 2 patients. No study employed tympanograms. The outcomes were positive in all three articles in terms of improvement of ORCHL[16,17], reduction of acute otitis media episodes[16,17], and OME resolution [18], though this last result was not statistically significant when compared to no treatment in a control group of cleft palate children. Besides primary outcomes, two papers [16,17] reported positive outcomes in subjective perceptual speech. A single study[17] reported outcomes in terms of hypernasality, which was improved in 3 out of 15 patients and unchanged in the remaining 12 patients. The speech evaluation was performed either: via an informal unspecific qualitative interview with parents and speech therapist [16] or via a non-further-specified speech therapist evaluation for hypernasality and general speech quality [17].

Finally, no reviewed study performed concurrent tympanostomy prior to or concurrent with adenoidectomy. A single study [18] performed tympanotomy in a number between 81 and 83 patients, but the timing of the procedure with regards to adenoidectomy was unclear.

Due to the heterogeneity and paucity of data, a meta-analysis could not be performed.

\section{CONCLUSIONS}

A major striking data is deriving from this systematic review: despite promising - albeit preliminary - results for adenoidectomy in treating middle ear disease in the $\mathrm{CP} \pm \mathrm{L}$ population, research in this field seems to have stopped in the mid-Seventies. This happened despite all articles included in this review report adenoidectomy (either with or tonsillectomy) as a valuable tool in treating middle ear disease in this population. A single article [18] failed to achieve statistical significance in its (albeit positive) results and suggested age as a major confounder for the results in this population. This objection has been indeed confirmed by studies confirming that middle ear disease in $\mathrm{CP} \pm \mathrm{L}$ children tends to improve with age[5].

It might be objected that the articles included in the systematic review lack a prospective design and their methodology - unremarkable in their historic context - might not hold up to today's technological standards. 
Nevertheless, upon rating and review, they all appear to have been conducted meticulously, and their content cannot be ignored.

Studies on the role of adenoidectomy in this population have been hampered by the constant fear that the procedure could have detrimental effects on the velopharyngeal function, often already impaired in this patient group[19]. This relatively common sequela of adenoidectomy has been linked to specific morphological characteristics [20,21], with a globally heterogeneous prevalence across studies. It has to be noted that even such a low incidence of velopharyngeal insufficiency in the selected studies appears too optimistic not to be related to a methodological bias in reporting complications. Such a hypothesis becomes even more realistic if we take a closer look at the speech evaluation methods used in the reviewed articles and to the scattered data reporting, as already described in the results. Furthermore, these evaluations nevertheless do not take into account the evolutions of the adenoidectomy technique in the endoscopic era. Not only power-assisted adenoidectomy has become a reliable tool in the general pediatric population [22], but its use in performing selective adenoidectomies has been widely demonstrated as a safe and reproducible tool also in the $\mathrm{CP} \pm \mathrm{L}$ population with no detrimental effect on speech [23] and velopharyngeal insufficiency[13,24]. Unfortunately, no study at present evaluated partial endoscopic adenoidectomy for middle ear disease in cleft patients.

It is also to be noted that current scientific reports confirm that adenoidectomy still represents a treatment choice in $\mathrm{CP} \pm \mathrm{L}$, despite its indications being presently limited to nasal breathing difficulties and obstructive sleep apnoea $[25,26]$.

Therefore if we take into account:

a) the preliminary good results on middle ear disease reported in the original, albeit outdated works on adenoidectomy in cleft children;

b) the introduction of less invasive modern endoscopic partial adenoidectomy techniques;

c) the efficacy of adenoidectomy in treating OME also in large scale meta-analysis; and

d) the routine use of adenoidectomy in the cleft population of other indications.

It comes as a surprise that no prospective studies on this subject have been proposed. The extremely wide use of tympanostomy as a first-line treatment for OME and ORCHL in this patient group represents a further direct consequence of the paucity of data on adenoidectomy and middle ear disease in the cleft population.

It has to be noted that this systematic review is limited in its strength as it included all article types, focusing on a wide range of middle ear conditions and with heterogeneous evaluation tools, but the lack of a significant bulk of literature on the subject made any further refinement impossible. Nevertheless, a call for stronger evidence on the subject emerges preponderantly. An unclear aspect of this review is worth examining indepth, i.e. the relationship in the $\mathrm{CP} \pm \mathrm{L}$ population between tympanostomy and adenoidectomy. As much as this interplay is important, only one reviewed study reported performing tympanotomy in nearly all patients, but with an unclear timing, while tympanostomy wasn't apparently performed on these patients. This overall management clashes with current trends in $\mathrm{CP} \pm \mathrm{L}$ patients with middle ear disease, so the results in these regards should be further put into context with future studies. Our literature review furthermore showed a complete lack of evidence in the use of tympanostomy tubes concurrent with adenoidectomy in the $\mathrm{CP} \pm \mathrm{L}$ population, as no studies addressing this particular subgroup was identified.

In the present context of middle ear disease in the cleft population, it would be unreasonable to suggest adenoidectomy as an alternative to tympanostomy. There is nevertheless a specific area of intervention where adenoidectomy could represent a powerful additional tool that requires urgent investigation. Cleft patients requiring re-tympanostomy (a population with a known higher risk of long-term otologic sequelae[5]) might benefit from concurrent tympanostomy and adenoidectomy to lower the risk of further tympanostomies. Prospective RCT of partial adenoidectomy in these patients would be feasible, ethical, and might hold great potential. Possible positive results might therefore help delineate a new and wider role for this old-fashioned technique. 


\section{DATA AVAILABILITY STATEMENT}

The data that support the findings of this study are available from the corresponding author upon reasonable request.

\section{REFERENCES}

1. Edetanlen EB, Saheeb BD. Otitis media with effusion in Nigerian children with cleft palate: incidence and risk factors. Br J Oral Maxillofac Surg 2019;57(1): 36-40.

2. Rynnel-Dagöö B, Lindberg K, Bagger-Sjöbäck D, Larson O. Middle ear disease in cleft palate children at three years of age. Int J Pediatr Otorhinolaryngol 1992;23(3): 201-209.

3. Tengroth B, Lohmander A, Hederstierna C. Hearing Thresholds in Young Children With Otitis Media With Effusion With and Without Cleft Palate. Cleft Palate Craniofac J 2020;57(5): 616-623.

4. Dhillon RS. The middle ear in cleft palate children pre and post palatal closure. J R Soc Med 1988;81(12): $710-713$.

5. Azman A, Manuel AM. Otological outcome in cleft lip and palate children with middle ear effusion. Int J Pediatr Otorhinolaryngol 2020;138: 10274.

6. Inoue M, Hirama M, Kobayashi S, et al. Long-term outcomes in children with and without cleft palate treated with tympanostomy for otitis media with effusion before the age of 2 years. Acta Otolaryngol 2020;140(12):982-989.

7. van den Aardweg MTA, Schilder AGM, Herkert E, Boonacker CWB, Rovers MM. Adenoidectomy for otitis media in children. Cochrane Database Syst Rev 2010;20(1):CD007810

8. Browning GG, Rovers MM, Williamson I, Lous J, Burton MJ. Grommets (ventilation tubes) for hearing loss associated with otitis media with effusion in children. Cochrane Database Syst Rev 2010;6(10):CD001801.

9. Venekamp RP, Mick P, Schilder AG, Nunez DA. Grommets (ventilation tubes) for recurrent acute otitis media in children. Cochrane Database Syst Rev 2018;5(5):CD012017.

10. NICE overview on suction diathermy adenoidectomy. Available: https://www.nice.org.uk/guidance/ipg328 Accessed November 30, 2020

11. Bellussi LM, Marchisio P, Materia E, Passàli FM. Clinical guideline on adenotonsillectomy: the Italian experience. Adv Otorhinolaryngol 2011;72:142-145.

12. Mushi E, Mahdi N, Upile N, et al. Velopharyngeal insufficiency in patients without a cleft palate: important considerations for the ENT surgeon. J Laryngol Otol 2020;134(3): 252-255.

13. Salna I, Jervis-Bardy J, Wabnitz D, et al. Partial Adenoidectomy in Patients With Palatal Abnormalities. J Craniofac Surg 2019;30(5):e454-e460.

14. Study Quality Assessment Tools. Available: https://www.nhlbi.nih.gov/health-topics/study-qualityassessment-tools Study Quality Assessment Tools. National Heart, Lung, and Blood Institute (NHLBI). https://www.nhlbi.nih.gov/health-topics/study-quality-assessment-tools. Accessed November 30, 2020

15. The Oxford Levels of Evidence 2. Oxford Centre for Evidence-Based Medicine. https://www.cebm.net/index.aspx?o=5653. Accessed November 30, 2020.

16. Chalat NI. Tonsillectomy, adenoidectomy and the cleft palate clinic. Laryngoscope 1965;75: 408-427.

17. Loeb WJ. Speech, hearing, and the cleft palate. Arch Otolaryngol 1964;79: 4-14.

18. Severeid LR. A longitudinal study of the efficacy of adenoidectomy in children with cleft palate and secretory otitis media. Trans Am Acad Ophthalmol Otolaryngol 1972;76(5):1319-1324. 
19. Witzel MA, Hampton Rich R, Margar-Bacal F, Cox C. Velopharyngeal insufficiency after adenoidectomy: an 8-year review. Int J Pediatr Otorhinolaryngol 1986;11(1):15-20.

20. Donnelly MJ. Hypernasality following adenoid removal. Ir J Med Sci 1994;163(5): 225-227.

21. Kerr WJ. The nasopharynx, face height, and overbite. Angle Orthod 1985;55(1):31-36.

22. Saibene AM, Rosso C, Pipolo C, et al. Endoscopic adenoidectomy: a systematic analysis of outcomes and complications in 1006 patients. Acta Otorhinolaryngol Ital 2020;40(1):79-86.

23. Abdel-Aziz M, Khalifa B, Shawky A, et al. Trans-oral endoscopic partial adenoidectomy does not worsen the speech after cleft palate repair. Braz J Otorhinolaryngol 2016;82(4):422-426.

24. Waselchuk E, Sidman JD, Lander T, Tibesar R, Roby BB. Sleep and Speech Outcomes After Superior Adenoidectomy in Children with Cleft Palate. Cureus 2018;10(1):e2097.

25. Muntz HR. Management of sleep apnea in the cleft population. Curr Opin Otolaryngol Head Neck Surg 2012;20(6): 518-521.

26. Abdel-Aziz M. The effectiveness of tonsillectomy and partial adenoidectomy on obstructive sleep apnea in cleft palate patients. Laryngoscope 2012;122(11): 2563-2567.

\section{TABLES}

Table 1- Characteristics of the included studies

\begin{tabular}{llllll}
\hline & Study type & OCEBM rating & NHI-SQAT rating & number of patients & Mean age \\
\hline Chalat, 1965 [16] & case series & 4 & $\mathrm{~F}$ & 38 & N/A \\
Loeb, 1964 [17] & case series & 4 & $\mathrm{~F}$ & 15 & N/A \\
Severeid, 1972 [18] & retrospective cohort study & 3 & $\mathrm{~F}$ & 83 & 11 \\
\hline
\end{tabular}

OCEBM: Oxford Centre for Evidence-Based Medicine; NI-SQAT: National Heart, Lung, and Blood Institute Study Quality Assessment Tools; $\mathrm{F}=$ fair; N/A= not available

Table 2- Data on cleft types, performed procedures, patients diagnosis, evaluation tools, and outcomes

\begin{tabular}{lllll}
\hline & Type of cleft(s) & Procedure performed & Indications to surgery & Evaluat \\
\hline Chalat, 1965 [16] & cleft lip and/or palate & A+T & ORCHL (38 patients), OMAR (35 patients) & clinical \\
Loeb, 1964 [17] & cleft palate & A or A+T & OMAR, worsening ORCHL & clinical \\
Severeid, 1972 [18] & cleft palate & A & OME & clinical \\
\hline
\end{tabular}

$\mathrm{A}=$ adenoidectomy $\mathrm{T}=$ tonsillectomy $\mathrm{A}+\mathrm{T}=$ adenotonsillectomy $; \mathrm{ORCHL}=$ OME-related conductive hearing losses; OME = otitis media with effusion; RAOM = recurrent acute otitis media; PTA = pure tone audiometry

Supplementary Material Captions

Supplementary Figure 1: PRISMA style flow diagram of studies selection through systematic review

Supplementary Table 1: Search strategy ad results for all consulted databases

Supplementary Table 2: List of articles excluded during the full-text evaluation 